\title{
DETERMINAÇÃO DE CAPACIDADE DE CAMPO IN SITU OU ATRAVÉS DE EQUAÇÕES DE REGRESSÃO' ${ }^{1}$
}

\author{
ADELAR JOSÉ FABIAN² e THEOPHILO BENEDICTO OTTONI FILHO
}

\begin{abstract}
RESUMO - Este trabalho foi desenvolvido com os objetivos de testar as equações globais obtidas por Macedo para determinar a capacidade de campo (CC) in situ em um solo Podzólico Vermelho-Amarelo; avaliar a câmara de fluxo desenvolvida por Fabian \& Ottoni Filho; e verificar se os valores de CC obtidos com esse equipamento se comparam aos determinados pelo método da Embrapa. Os testes foram realizados em 1994, num Podzólico Vermelho-Amarelo, em Itaguaí, RJ. A motivação desta comparação é o fato de o movimento lateral da água ser praticamente eliminado dentro da câmara. Confirmou-se que os valores medidos de $\mathrm{CC}$ em tal equipamento reproduziram as determinações de CC obtidas pelo método da Embrapa. Como a área da câmara é de $0,50 \mathrm{~m}^{2}$, o resultado sugere a possibilidade de reduzir a dimensão dos tabuleiros de inundação. Foram também validadas as equações globais de regressão para determinar a CC a partir de porcentagens texturais e de matéria orgânica, ou a partir da microporosidade (umidade a $60 \mathrm{~cm}$ de tensão).
\end{abstract}

Termos para indexação: retenção de água no solo, umidade do solo, equipamento de drenagem, drenagem interna, medição de pedofunções.

\section{DETERMINATION OF FIELD CAPACITY IN SITU OR BY REGRESSION EQUATIONS}

\begin{abstract}
This work was developed in order to test the global equations obtained by Macedo to determine the in situ field capacity (FC) in a Typic Kanhapludalf soil; to evaluate the flux chamber developed by Fabian \& Ottoni Filho; and to verify how the FC values obtained with this equipment compare to the values determined through the method of Embrapa. The tests took place in 1994, in a Typic Kanhapludalf soil, in Itaguaí, RJ, Brazil. The motivation for such a comparison is the fact that water lateral movement is practically eliminated inside the chamber. It was confirmed that the FC values measured in such an equipment, reproduced the FC determinations obtained through the methodology of Embrapa. As the area of the chamber is $0.50 \mathrm{~m}^{2}$, the result suggests the possibility of decrease in the flooding basin dimension. The regression global equations obtained by Macedo were validated, determining FC based on textural and organic matter percentages, or based on microporosity ( $60 \mathrm{~cm}$ tensionsoil moisture).
\end{abstract}

Index terms: soil water retention, soil moisture, drainage equipment, internal drainage, pedofunctions measurement.

\section{INTRODUÇÃO}

A quantidade de água que um perfil de terreno sem vegetação e evaporação retém contra a ação da

\footnotetext{
${ }^{1}$ Aceito para publicação em 13 de julho de 1999 .

Extraído da tese de mestrado do primeiro autor, apresentada à Universidade Federal Rural do Rio de Janeiro (UFRRJ).

${ }^{2}$ Eng. Agrôn., M.Sc., Escola Agrotécnica Federal de Cuiabá, Caixa Postal 67, CEP 78106-000 São Vicente da Serra, MT.

${ }^{3}$ Eng. Civil, Ph.D., Dep. de Solos, UFRRJ, CEP 23851-970 Seropédica, RJ. E-mail: dhs@civil.ee.ufrj.br
}

gravidade, após plenamente inundado e deixado drenar livremente por uns poucos dias (um a quatro dias), em condições de campo, determina o volume máximo aproximado de água que um solo bem drenado pode armazenar por longos períodos sem evapotranspiração. Esta umidade é chamada capacidade de campo do solo, segundo definição dada por Veihmeyer \& Hendrickson (1931).

Alguns estudos mostram que a umidade do solo na capacidade de campo não está totalmente em equilíbrio e que o movimento da água pode continuar por vários dias ou mesmo meses (Hillel, 1980; Reichardt, 
1988). Inúmeros trabalhos realizados em laboratório com amostras deformadas e indeformadas objetivaram definir uma determinada tensão de água (potencial matricial de água no solo), geralmente 33 e $10 \mathrm{kPa}$, que fosse correspondente à umidade retida na capacidade de campo medida in situ (Lund et al., citados por Swain \& Scotter, 1988). Porém, ainda não há uma posição consensual sobre a correta tensão associada à capacidade de campo em cada tipo de solo, e sobre o tempo de drenagem para atingir o equilíbrio (Salter \& Williams, 1965; Oliveira \& Melo, 1971; Reichardt, 1988).

A capacidade de campo pode ser influenciada pela textura e estrutura do solo, teor de matéria orgânica, seqüência dos horizontes pedogenéticos e gradiente textural entre os horizontes, bem como pelo teor inicial de umidade do solo e lâmina d'água aplicada (Marshall, citado por Salter \& Williams, 1965; Oliveira \& Queiroz, 1975; Poulovassilis, 1983; Boédt \& Verheye, 1985).

A determinação da capacidade de campo in situ, segundo Embrapa (1979), é realizada num tabuleiro de 1,0 m x 1,0 m, onde se aplica uma lâmina de água suficiente para saturar o perfil até a profundidade desejada. Esta lâmina é obtida pela diferença entre a porosidade e a umidade inicial, integrada ao longo do perfil, acrescentando-se uma porcentagem relativa às perdas laterais causadas pelo fluxo horizontal. Tais perdas são induzidas pelos gradientes horizontais de pressão entre o solo úmido sob o tabuleiro e o terreno mais seco circundante. Quanto maior a dimensão do tabuleiro, menor a influência desse fluxo de perda horizontal na estabilização do perfil de umidade decorrente da drenagem interna (Hillel, 1980). Nessas experimentações de capacidade de campo in situ é comum a utilização de tabuleiros maiores que os preconizados pela Embrapa (Kiehl, 1979; Schub et al., 1988; Paige \& Hillel, 1993). Portanto, uma questão metodológica relevante é a definição do tabuleiro mínimo do experimento.

Fabian \& Ottoni Filho (1997) desenvolveram um equipamento denominado câmara de fluxo, que consiste num cilindro metálico de $80 \mathrm{~cm}$ de diâmetro por $80 \mathrm{~cm}$ de altura e que pode ser totalmente cravado no terreno por pressão hidráulica, sem necessidade de nenhuma perturbação na estrutura do solo. $\mathrm{O}$ equipamento é particularmente indicado para pes- quisas in situ envolvendo processos de transferência verticais, como é a drenagem interna após a plena inundação do perfil. A utilização desse equipamento na determinação in situ da capacidade de campo, apresenta a vantagem de eliminar os fluxos horizontais de perda d'água das camadas superiores do perfil.

A determinação da capacidade de campo de forma indireta, principalmente a partir de propriedades físicas do solo, é objeto de várias pesquisas. Isto se explica pelas dificuldades práticas do procedimento in situ. Hollis et al. (1977) observaram uma correlação entre os conteúdos de argila, silte, silte + argila e carbono orgânico, e a umidade volumétrica na fração fina do solo na tensão de $5 \mathrm{kPa}$. Neste estudo, o carbono orgânico demonstrou ser o mais importante parâmetro de correlação, seguido pela argila. Já no trabalho de Petersen et al. (1968), o carbono orgânico não demonstrou nenhuma relação com a retenção de umidade a $33 \mathrm{kPa}$. Rivers \& Shipp (1978), Gupta \& Larson (1979), Rawls et al. (1982), Ahuja et al. (1985), Saxton et al. (1986) e Arruda et al. (1987) trabalharam com funções matemáticas, denominadas pedofunções, relacionando a retenção de água a diversas tensões, com a textura, densidade do solo e teor de matéria orgânica. Vale ressaltar que nos perfis arenosos os valores da capacidade de campo em laboratório (a partir de um nível de tensão preestabelecido) podem ser altamente dependentes do teor de argila (Petersen et al., 1968). Oliveira \& Melo (1971) verificaram que os valores de equivalente de umidade obtidos com amostras saturadas e centrifugadas a uma rotação de 2.440 rpm na centrífuga de Briggs \& McLane não foram adequados para expressar os valores de capacidade de campo in situ, especialmente em solos de textura leve. Já a microporosidade determinada pelo método da mesa de tensão correspondeu, naquele estudo, a valores muito próximos da capacidade de campo determinada in situ. Mais recentemente, visando determinar a curva característica de retenção hídrica dos solos, têm sido testadas pedofunções que admitem, também, como variáveis de entrada, um ou dois valores de umidade correspondentes a níveis preestabelecidos de tensão (Williams et al., 1992; Basted et al., 1998).

Macedo (1991) conduziu estudos para estimar a retenção hídrica por correlação linear múltipla, em 
terrenos Podzólicos Vermelho-Amarelos no município de Itaguaí, RJ, a partir do teor de matéria orgânica e de dados físicos que normalmente são encontrados nas descrições pedológicas. Os resultados mostraram que textura e matéria orgânica foram os fatores primordiais quanto à caracterização da retenção de água naqueles solos. A partir daí, foram desenvolvidas equações globais de regressão que calcularam suficientemente bem os dados médios espaciais de retenção hídrica na área total estudada, na capacidade de campo in situ e em todas as faixas de tensão. As equações foram originalmente calibradas com dados de quatro solos Podzólicos VermelhoAmarelos, de forma que uma extrapolação para demais classes de solos deve ser evitada em princípio. Na realidade, Macedo (1991) sugere que tais equações devam ser aplicadas quando se deseja valores médios de retentividade hídrica em áreas com várias unidades desta classe, fazendo restrições ao seu uso numa única unidade. Entretanto, segundo o autor, isto é possível caso seja um Podzólico VermelhoAmarelo com alto teor de areia, nas classes texturais areia, areia-franca ou franco-arenosa. Portanto, uma questão relevante no método indireto de determinação de capacidade de campo ou retenção de umidade é a delimitação das fronteiras de aplicação de suas pedofunções.

Os objetivos deste trabalho foram: testar as equações globais obtidas por Macedo (1991), para determinar a capacidade de campo no solo Podzólico Vermelho-Amarelo do trabalho de Fabian \& Ottoni Filho (1997); avaliar a câmara de fluxo desenvolvida por Fabian \& Ottoni Filho (1997); e verificar se os valores de capacidade de campo obtidos com esse equipamento se comparam aos determinados pelo método da Embrapa (1979).

\section{MATERIAL E MÉTODOS}

A determinação da capacidade de campo in situ ocorreu de duas maneiras, no mesmo solo do trabalho de Fabian \& Ottoni Filho (1997): a) pelo método direto (Embrapa, 1979), em tabuleiros quadrados de inundação, de $1 \mathrm{~m}$ x $1 \mathrm{~m}$, localizados a cerca de $5 \mathrm{~m}$ dos locais onde foi instalada a câmara de fluxo, totalizando quatro ensaios; b) utilizando a câmara de fluxo, após os testes de infiltração nela (Fabian \& Ottoni Filho, 1997), também em quatro ensaios.
Em ambas as técnicas foi aplicada uma lâmina de inundação durante oito horas, suficiente para saturar o perfil até pelo menos $100 \mathrm{~cm}$ de profundidade. A aplicação da água foi simultânea na câmara e no tabuleiro contíguo. Após a água se infiltrar totalmente, os terrenos da câmara e tabuleiro, bem como a área circundante a esses terrenos, foram cobertos com lona de polietileno de $4 \mathrm{~m} \mathrm{x} 4 \mathrm{~m}$ para evitar perdas por evaporação, ou acréscimos pela chuva direta ou pela percolação horizontal perimetral ao tabuleiro ou câmara, tendo sido a lona enterrada verticalmente $50 \mathrm{~cm}$ em suas bordas.

A umidade volumétrica foi monitorada nas profundidades de $15,30,45$ e $60 \mathrm{~cm}$, no centro da câmara e do tabuleiro, simultaneamente. Ela foi obtida com uma sonda de nêutrons (Troxler 4300) previamente calibrada para o terreno estudado (Fabian \& Ottoni Filho, 1997). Para acesso da sonda, introduziu-se um tubo de alumínio de $5 \mathrm{~cm}$ de diâmetro. A umidade volumétrica foi determinada antes de iniciar a inundação, imediatamente depois de toda a água se infiltrar, e 17, 24, 41, 48, 72 e 96 horas após. A capacidade de campo, por profundidade de medição em cada área de inundação, foi considerada como o valor da última determinação de umidade.

Após o término do teste para capacidade de campo, foram coletadas amostras de solo abrindo-se trincheiras ao lado dos tubos de acesso da sonda de nêutrons, nos quatro tabuleiros e nas quatro posições da câmara. Foram retiradas amostras nas mesmas profundidades onde ocorreram as leituras da umidade. Foram monitorados o teor de matéria orgânica e as mesmas propriedades físicas do trabalho de Fabian \& Ottoni Filho (1997), segundo metodologia idêntica.

Visando determinar a capacidade de campo por regressão, utilizaram-se as equações globais desenvolvidas por Macedo (1991) a partir das porcentagens texturais, teor de matéria orgânica e microporosidade (umidade volumétrica na tensão de $60 \mathrm{~cm}$ ). A capacidade de campo (volumétrica) foi calculada a partir das seguintes equações:

a) com cinco variáveis independentes:

$\% \mathrm{CC}=\%$ argila $\mathrm{x}(-0,01)+\%$ areia $\mathrm{x}(-0,37)+\%$ M.O. $\mathrm{x}$ $(1,36)+\%$ areia grossa $\mathrm{x}(-0,02)+\%$ microporosidade $\mathrm{x}$ $(0,19)+42,20$ (equação 1)

b) com três variáveis independentes:

$\% \mathrm{CC}=\%$ argila $\mathrm{x}(0,05)+\%$ areia $\mathrm{x}(-0,45)+\%$ M.O. $\mathrm{x}$ $(1,80)+49,39$ (equação 2)

c) com uma variável independente:

$\% \mathrm{CC}=\%$ microporosidade $\mathrm{x}(0,80)+2,32 \quad$ (equação 3$)$

Para comparar os valores calculados de capacidade de campo com os valores medidos, foram utilizados os testes 
qui-quadrado e t de Student (Pimentel-Gomes, 1993). O teste qui-quadrado verifica se os valores calculados são, um a um, estatisticamente idênticos aos correspondentes valores medidos. O teste $t$ de Student verifica a hipótese nula de que a média de um conjunto de valores calculados em cada equação de regressão seja estatisticamente igual à capacidade de campo média mensurada.

\section{RESULTADOS E DISCUSSÃO}

As estatísticas básicas da distribuição espacial das variáveis de solo são mostradas na Tabela 1. A densidade das partículas apresentou valor quase constante de $2,65 \mathrm{~g} \mathrm{~cm}^{-3}$ ao longo do perfil. Este número corresponde à densidade das partículas de quartzo. Isto se deve à predominância de areia na fração fina do solo. A textura do solo varia de argiloarenosa a areia-franca. A porosidade total (VTP) é em torno de $0,34 \mathrm{dm}^{3} \mathrm{dm}^{-3}$. A argila foi a que apresentou o maior coeficiente de variação (58\%), seguida pelo teor de matéria orgânica. Este último manteve-se com valores inferiores a $1,0.10^{-2} \mathrm{~kg} \mathrm{~kg}^{-1} \mathrm{em}$ todas as amostras. A capacidade de campo (CC) medida apresentou coeficiente de variação médio $(23,5 \%)$, segundo a classificação de Warrick \& Nielsen (1980). Os valores médios de CC foram crescentes com a profundidade, variando de 0,16 a $0,26 \mathrm{dm} \mathrm{dm}^{-3}$, com o mesmo sentido de crescimento da porcentagem de argila ao longo do perfil. A microporosidade foi quase sempre muito próxima da CC.

Espera-se que em condições de campo, o processo da drenagem interna, após a inundação prolongada do terreno, seja um fenômeno de transferência vertical. Essa verticalidade de fluxo é garantida dentro da câmara de fluxo até a profundidade de $70 \mathrm{~cm}$ (Fabian \& Ottoni Filho, 1997), mas não é garantida no tabuleiro, em virtude dos gradientes horizontais de pressão. Logo, a umidade estabilizada no experimento dentro da câmara é a que, preferencialmente, deve ser tomada como padrão de medida da capacidade de campo. Para testar se os valores de retenção hídrica estabilizada nos perfis de solo no tabuleiro correspondem à capacidade de campo padrão, ou seja, à umidade estabilizada na câmara, foi aplicado o teste qui-quadrado separadamente aos 16 (4 x 4) dados de umidade da câmara e do tabuleiro. Procurouse comprovar, pelo teste qui-quadrado, se as 16 umi-

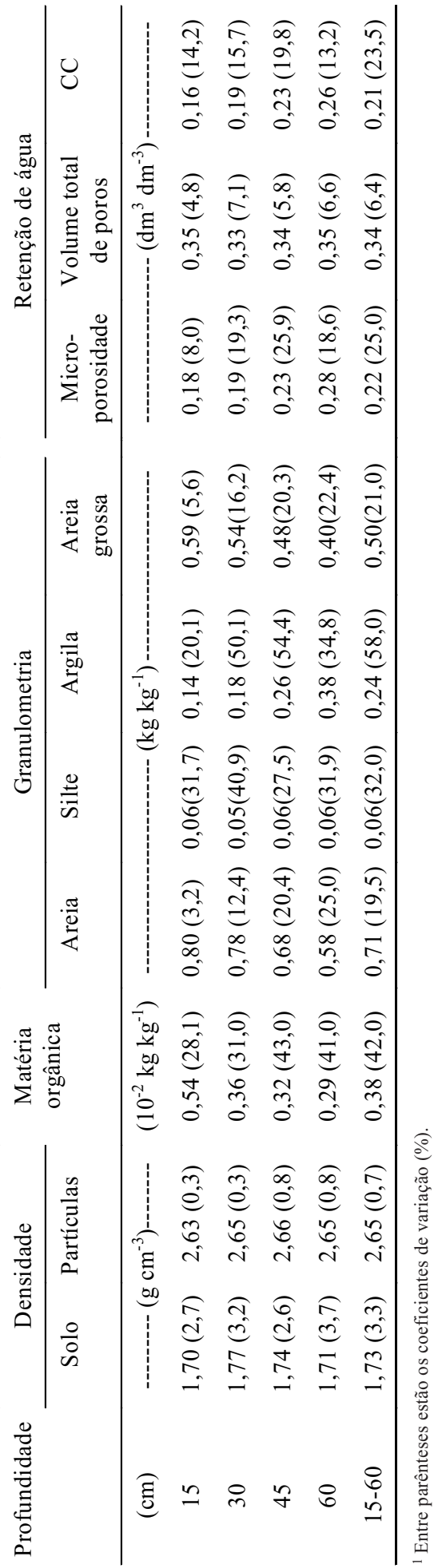


dades calculadas em cada uma das três pedofunções propostas (equações 1 a 3 ) se aproximam adequadamente das umidades estabilizadas que foram mensuradas na câmara e tabuleiro (Tabela 2).

$\mathrm{O}$ teste qui-quadrado indicou que não houve diferença significativa (a 5\% e 1\%) entre os 16 valores calculados por qualquer das três equações e os 16 valores mensurados, tanto na câmara como no tabuleiro. Daí, por transitividade, conclui-se que as umidades estabilizadas mensuradas nos tabuleiros podem ser tomadas como realizações da capacidade de campo padrão; constata-se, no experimento, que o fluxo de redistribuição lateral para fora do tabuleiro não acarreta mudança significativa na umidade estabilizada do processo de drenagem interna puramente vertical, umidade essa que corresponde ao valor da capacidade de campo in situ. Portanto, pode-se considerar que neste estudo há 32 realizações de capacidade de campo in situ, 16 dentro da câmara e 16 sob o tabuleiro.

Uma questão prática que é relevante nos testes de campo é a avaliação da área mínima para aplicação de água, tendo em vista as dificuldades de seu armazenamento e transporte. A análise do parágrafo anterior indicou que o tabuleiro de $1,0 \mathrm{~m}^{2}$ recomendado pela Embrapa foi de tamanho adequado para simular a inundação (que deveria ser de área infinita, teoricamente) de um experimento de capacidade de campo. Deve-se levar em conta, entretanto, que o terreno é de textura arenosa, o que deve minimizar as fugas laterais de percolação para fora do tabuleiro. Portanto, recomenda-se a repetição da metodologia deste trabalho (ou de outra metodologia equivalente) para diversos solos, visando a uma avaliação mais completa da influência do tamanho do tabuleiro no comportamento da umidade estabilizada no experi-

TABELA2. Valores observados e calculados de capacidade de campo, nas diferentes posições da câmara de fluxo e nos tabuleiros de inundação.

\begin{tabular}{|c|c|c|c|c|c|c|c|c|c|}
\hline \multirow[t]{4}{*}{ Local } & \multirow{4}{*}{$\begin{array}{l}\text { Profun- } \\
\text { didade }\end{array}$} & \multicolumn{8}{|c|}{ Capacidade de campo } \\
\hline & & \multicolumn{2}{|c|}{ Medida } & \multicolumn{6}{|c|}{ Calculada } \\
\hline & & \multirow[t]{2}{*}{ Câmara } & \multirow[t]{2}{*}{ Tabuleiro } & \multicolumn{2}{|c|}{ Equação 1} & \multicolumn{2}{|c|}{ Equação 2} & \multicolumn{2}{|c|}{ Equação 3} \\
\hline & & & & Câmara & Tabuleiro & Câmara & Tabuleiro & Câmara & Tabuleiro \\
\hline & $(\mathrm{cm})$ & 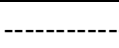 & $-\cdots$ & 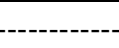 & $-\left(\mathrm{dm}^{3} \mathrm{~d}_{1}\right.$ & $-3)$ & & & 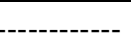 \\
\hline \multirow[t]{4}{*}{1} & 15 & 0,169 & 0,169 & 0,164 & 0,160 & 0,159 & 0,159 & 0,175 & 0,167 \\
\hline & 30 & 0,229 & 0,227 & 0,251 & 0,198 & 0,257 & 0,204 & 0,231 & 0,175 \\
\hline & 45 & 0,275 & 0,281 & 0,287 & 0,254 & 0,294 & 0,274 & 0,271 & 0,191 \\
\hline & 60 & 0,294 & 0,290 & 0,315 & 0,288 & 0,327 & 0,299 & 0,279 & 0,263 \\
\hline \multirow[t]{4}{*}{2} & 15 & 0,132 & 0,155 & 0,130 & 0,156 & 0,125 & 0,152 & 0,151 & 0,167 \\
\hline & 30 & 0,154 & 0,183 & 0,121 & 0,145 & 0,113 & 0,139 & 0,151 & 0,159 \\
\hline & 45 & 0,178 & 0,211 & 0,140 & 0,189 & 0,128 & 0,189 & 0,183 & 0,183 \\
\hline & 60 & 0,214 & 0,242 & 0,174 & 0,198 & 0,171 & 0,193 & 0,183 & 0,231 \\
\hline \multirow[t]{4}{*}{3} & 15 & 0,130 & 0,160 & 0,145 & 0,156 & 0,139 & 0,153 & 0,159 & 0,167 \\
\hline & 30 & 0,169 & 0,200 & 0,133 & 0,159 & 0,128 & 0,154 & 0,143 & 0,207 \\
\hline & 45 & 0,188 & 0,229 & 0,137 & 0,224 & 0,130 & 0,212 & 0,151 & 0,263 \\
\hline & 60 & 0,220 & 0,250 & 0,199 & 0,292 & 0,196 & 0,302 & 0,207 & 0,271 \\
\hline \multirow[t]{4}{*}{4} & 15 & 0,156 & 0,201 & 0,158 & 0,173 & 0,154 & 0,168 & 0,167 & 0,191 \\
\hline & 30 & 0,168 & 0,228 & 0,143 & 0,188 & 0,134 & 0,190 & 0,167 & 0,191 \\
\hline & 45 & 0,185 & 0,283 & 0,136 & 0,265 & 0,129 & 0,275 & 0,159 & 0,247 \\
\hline & 60 & 0,242 & 0,300 & 0,216 & 0,342 & 0,219 & 0,359 & 0,215 & 0,303 \\
\hline
\end{tabular}


mento in situ. O que seria desejável é que as inundações fossem realizadas em tabuleiros ainda menores do que $1,0 \mathrm{~m}^{2}$. O fato de a câmara ter $0,50 \mathrm{~m}^{2}$ sugere a possibilidade de redução no tamanho dos tabuleiros. Isto talvez possa ocorrer em muitos casos, pois as fugas laterais que inevitavelmente ocorrem durante a infiltração da lâmina aplicada no tabuleiro fazem diminuir os gradientes horizontais de pressão durante a fase de drenagem interna.

Deve-se enfatizar que, por causa do processo mais complexo de instalação da câmara de fluxo, não se está propondo a substituição do tabuleiro pela câmara, na metodologia in situ de determinação de CC. Entretanto, os fluxos verticais de água podem ser mais realisticamente estudados dentro da câmara, o que motiva seu uso em experimentos de pesquisa e de comprovação de validade de metodologias de campo, como foi feito neste trabalho com a CC e, no trabalho de Fabian \& Ottoni Filho (1997), com a infiltração. Na realidade, o mesmo experimento de aplicação de água na câmara permitiu a avaliação de ambas as metodologias in situ, de capacidade de campo e de infiltração.

As curvas de drenagem interna representam a variação temporal da umidade a partir do término da infiltração nas áreas inundadas. A Fig. 1 mostra as curvas médias por profundidade, utilizando todas as umidades mensuradas nas quatro posições da câmara e nos tabuleiros. Praticamente não se observam variações de umidade após 48 horas do término da infiltração, o que é concordante com a clássica definição de capacidade de campo dada por Veihmeyer \& Hendrickson (1931). O tempo zero foi o momento no qual toda lâmina d'água aplicada na superfície do solo infiltrou-se.

A Fig. 2 mostra os 32 pontos do valor calculado (equação 3) versus o observado de capacidade de campo. Se não houvesse nenhum erro de estimativa, os pontos estariam todos sobre a linha 1:1 indicada. Uma questão relevante é saber se esses erros introduzem variações significativas na estimativa da CC média no perfil, que foi de $0,21 \mathrm{dm}^{3} \mathrm{dm}^{-3}$ (Tabela 1 ). Visando responder a esta pergunta foi aplicado o teste $t$ de Student aos 32 pares de dados observados versus os calculados em cada uma das três equações de regressão propostas. O resultado (Tabela 3) é que a CC média foi estimada pelas três equações sem diferenças significativas em relação à média observada. O menor erro relativo $(5,2 \%)$ foi com respeito à equação 3 , que foi a que teve apenas a microporosidade como variável de entrada.

O resultado acima confirma a constatação da literatura (Oliveira \& Melo, 1971; Rivers \& Shipp, 1978; Macedo, 1991), de que a microporosidade pode ser

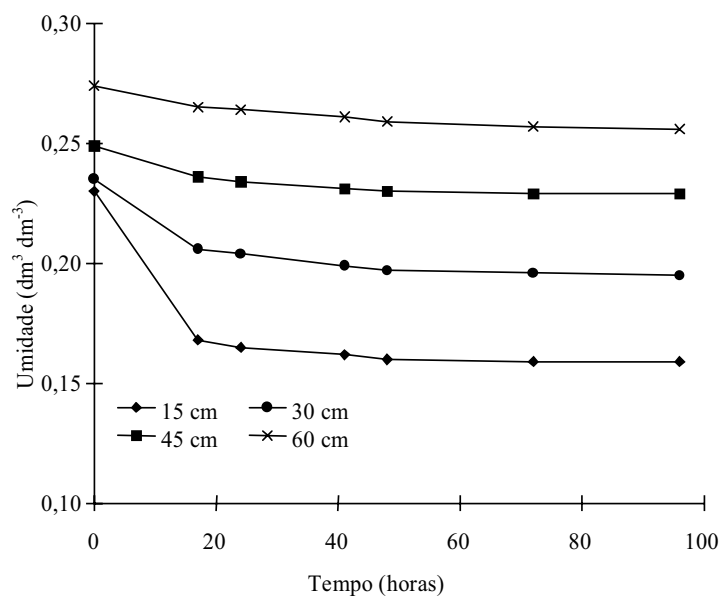

FIG. 1. Curvas de drenagem interna: média espacial por profundidade.

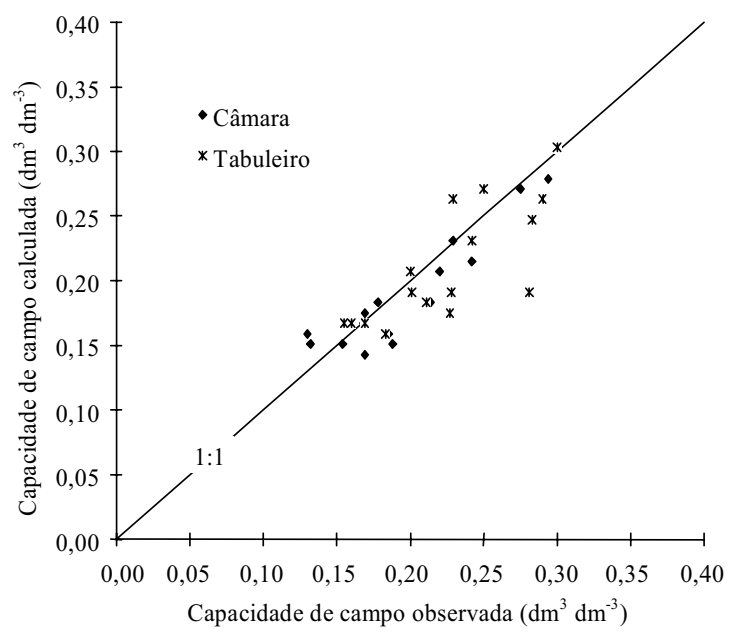

FIG. 2. Capacidade de campo observada e calculada pela equação 3, no tabuleiro e câmara. 
TABELA 3. Aplicação do teste t de Student na comparação dos valores médios observado e calculado de capacidade de campo (CC). São também indicadas as estatísticas calculadas e tabeladas.

\begin{tabular}{cccccc}
\hline Equação & $\begin{array}{c}\mathrm{CC} \\
\text { calculada }\end{array}$ & $\mathrm{ER}^{1}$ & \multicolumn{3}{c}{ Teste $\mathrm{t}$} \\
\cline { 5 - 6 } & & $\mathrm{t}$ calculado & $\begin{array}{c}\text { t tabelado } \\
(5 \%)\end{array}$ & $\begin{array}{c}\mathrm{t} \text { tabelado } \\
(1 \%)\end{array}$ \\
\hline & $\left(\mathrm{dm}^{3} \mathrm{dm}^{-3}\right)$ & $(\%)$ & & & 2,75 \\
1 & 0,195 & 7,1 & 1,08 & 2,04 & 2,75 \\
2 & 0,194 & 7,6 & 1,07 & 2,04 & 2,75 \\
\hline
\end{tabular}

${ }^{1}$ Porcentagem de erro relativo, em relação ao valor médio observado de $0,21 \mathrm{dm}^{3} \mathrm{dm}^{-3}$.

utilizada como estimador, em solos arenosos, da capacidade de campo in situ. Na realidade, mais precisamente, pode-se dizer que a capacidade de campo deve ser estimada por uma função da microporosidade, para esses solos. Entretanto, é aconselhável que se delineie o grau de generalidade desta regra.

Aplicando-se a equação 3 aos dados de microporosidade do trabalho de Oliveira \& Melo (1971), a capacidade de campo calculada seria de $0,192,0,182$, 0,252 e $0,254 \mathrm{dm}^{3} \mathrm{dm}^{-3}$, para os quatro horizontes estudados do solo. A capacidade de campo mensurada in situ foi, correspondentemente, de 0,207, 0,190, 0,242 e $0,251 \mathrm{dm}^{3} \mathrm{dm}^{-3}$. Portanto, a porcentagem média de erro relativo nesse caso foi de apenas $4,2 \%$. A textura variou de arenosa, no horizonte superficial $(0-17 \mathrm{~cm})$, até franco-argilo-arenosa $(60-100 \mathrm{~cm})$.

Deve-se enfatizar que, como as equações aqui utilizadas foram calibradas e validadas apenas em solos Podzólicos Vermelho-Amarelos com alto teor de areia e não alto teor de argila (pelo menos com textura franco-argilo-arenosa ou franco-arenosa), não se indica, a princípio, sua aplicação em outras classes de solo.

\section{CONCLUSÕES}

1. A câmara de fluxo reproduz adequadamente os fenômenos de transferência hídrica vertical.

2. Os valores da capacidade de campo obtidos com o uso da câmara de fluxo são equivalentes aos obtidos pelo método da Embrapa; o resultado sugere que o tamanho do tabuleiro preconizado pela Embrapa (1,00 $\mathrm{m}^{2}$ de área) pode ser reduzido.
3. A capacidade de campo in situ de solos Podzólicos Vermelho-Amarelos arenosos é determinada adequadamente pelas equações globais de regressão de Macedo.

\section{REFERÊNCIAS}

AHUJA, L.R.; NAMEY, J.W.; WILLIAMS, R.D. Estimating soil water characteristics from simpler properties or limited data. Soil Science Society of America. Journal, Madison, v.49, p.1100-1105, 1985.

ARRUDA, F.B.; ZULLO JÚNIOR, J.; OLIVEIRA, J.B. Parâmetros de solo para o cálculo da água disponível com base na textura do solo. Revista Brasileira de Ciência do Solo, Campinas, v.11, p.11-15, 1987.

BASTED, G.; BRUAND, A.; VOLTZ, M.; BORNAND, M.; QUETIN, P. Prediction of water retention properties: performance of available pedotransfer functions and development of new approaches. In: CONGRES MONDIAL DE SCIENCE DU SOL, 16., 1998, Montpellier. Résumés. Montpellier : ISSS-AISS-IBG-SICS/AFES, 1998. v.1, p.22. Trabalho apresentado na íntegra na forma de pôster.

BOÉDT, L.; VERHEYE, W. Evaluation of profile available water capacity. 1. The conceptual approach. Pedologie, Ghent, v.35, p.55-65, 1985.

EMBRAPA. Serviço Nacional de Levantamento e Conservação de Solos (Rio de Janeiro, RJ). Manual de métodos de análise de solo. Rio de Janeiro, 1979. Não paginado.

FABIAN, A.J.; OTTONI FILHO, T.B. Determinação de curvas de infiltração usando uma câmara de fluxo.

Pesq. agropec. bras., Brasília, v.35, n.5, p.1029-1036, maio 2000 
Revista Brasileira de Ciência do Solo, Campinas, v.21, p.325-333, 1997.

GUPTA, S.C.; LARSON, W.E. Estimating soil water retention characteristics from particle size distribution, organic matter content, and bulk density. Water Resources Research, Washington, v.20, p.1633-1635, 1979.

HILLEL, D. Internal drainage and redistribution following infiltration. In: HILLEL, D. (Ed.). Applications of soil physics. New York : Academic, 1980. p.50-72.

HOLLIS, J.M.; JONES, R.J.A.; PALMER, R.C. The effects of organic mater and particle size on the water retention properties of some soils in the west midlands of England. Geoderma, Amsterdam, v.17, p.225-238, 1977.

KIEHL, E.J. Manual de edafologia. São Paulo : Agronômica Ceres, 1979. Cap.13, 264p.

MACEDO, J.R. Determinação de retenção hídrica por correlação múltipla e de variabilidade espacial em solos podzólicos de Seropédica, RJ. Rio de Janeiro : UFRRJ, 1991. 174p. Dissertação de Mestrado.

OLIVEIRA, L.B.; MELO, V. Estudo da disponibilidade de água em um solo da estação experimental de Itapirema, Pernambuco. Pesquisa Agropecuária Brasileira, Série Agronomia, Rio de Janeiro, v.6, p.31-37, 1971.

OLIVEIRA, L.B.; QUEIROZ, E.N. Curvas características de retenção de umidade de solos do Nordeste do Brasil. Pesquisa Agropecuária Brasileira, Série Agronomia, Rio de Janeiro, v.10, p.69-75, 1975.

PAIGE, G.B.; HILLEL, D. Comparison of three methods for assessing soil hydraulic properties. Soil Science, Baltimore, v.155, p.175-189, 1993.

PETERSEN, G.W.; CUNNINGHAM, R.L.; MATELSKI, R.P. Moisture characteristics of Pennsylvania soils. I. Moisture retention as related to texture. Soil Science Society of America. Proceedings, Madison, v.32, p.271-275, 1968.

PIMENTEL-GOMES, F. Curso de estatística experimental. 4.ed. Piracicaba : Nobel, 1993. 430p.

POULOVASSILIS, A. The influence of the initial water content on the redistribution of soil water after infiltration. Soil Science, Baltimore, v.135, p.275281,1983 .

RAWLS, W.J.; BRAKENSIEK, D.L.; SAXTON, K.E. Estimation of soil water properties. Transactions of the ASAE, Saint Joseph, v.25, p.1316-1320, 1982.

REICHARDT, K. Capacidade de campo. Revista Brasileira de Ciência do Solo, Campinas, v.12, p.211216,1988 .

RIVERS, E.D.; SHIPP, R.F. Soil water retention as related to particle size in selected sands and loamy sands. Soil Science, Baltimore, v.126, p.94-100, 1978.

SALTER, P.J.; WILLIAMS, J.B. The influence of texture on the moisture characteristics of soils. I. A critical comparison of techniques for determining the available water capacity and moisture characteristic curve of a soil. Journal of Soil Science, Oxford,v.16, p.1-15, 1965.

SAXTON, K.E.; RAWLS, W.J.; ROMBERGER, J.S.; PADENDICK, R.I. Estimating generalized soil-water characteristics from texture. Soil Science Society of America. Journal, Madison, v.50, p.1031-1036, 1986

SCHUB, W.M.; CLINE, R.L.; SWEENEY, M.D Comparison of a laboratory procedure and a textural model for predicting in situ soil water retention. Soil Science Society of America. Journal, Madison, v.52, p.1218-1227, 1988 .

SWAIN, D.J.; SCOTTER, D.R. Hydraulic properties and field capacity of Himatangi sand. New Zealand Journal of Experimental Agriculture, Lower Hutt, v.16, p.367-374, 1988.

VEIHMEYER, F.J.; HENDRICKSON, A.H. The moisture equivalent as a measure of the field capacity of soil Soil Science, Baltimore, v.32, p.181-193, 1931.

WARRICK, A.W.; NIELSEN, D.R. Spatial variability of soil physical properties in the field. In: HILLEL, D. (Ed.). Applications of soil physics. New York : Academic, 1980. p.319-344.

WILLIAMS, R.D.; AHUJA, L.R.; NAMEY, J.W Comparison of methods to estimate soil water characteristics from soil texture, bulk density, and limited data. Soil Science, Baltimore, v.153, p.172184, 1992. 